\title{
SPH Simulations of Galaxy Cluster Mergers
}

\author{
Motokazu Takizawa \\ Department of Physics, Yamagata University, Kojirakawa-machi 1-4-12, \\ Yamagata 990-8560, Japan
}

\begin{abstract}
We present our results of SPH simulations of cluster mergers. We investigate the temperature evolution explicitly considering the relaxation process between ions and electrons. The electron temperature distributions are significantly different than those of ions in the later stages of mergers. We also investigate evolution of non-thermal emission from relativistic electrons accelerated around shock fronts. Hard X-ray is luminous only while signatures of merging events are clearly seen in the thermal intracluster medium. On the other hand, extreme ultraviolet emission is still luminous after the system has relaxed.
\end{abstract}

\section{Introduction}

Some clusters of galaxies show peculiar density and temperature distribution in intracluster medium. It is believed that such clusters are now being formed through mergers. Indeed, such a situation is expected from the hierarchical structure formation scenario. In this paper, we present our results of N-body + SPH simulations of cluster mergers. (Takizawa 1999, 2000). We also investigate evolution of non-thermal emission from relativistic electrons accelerated around shock fronts (Takizawa \& Naito 2000).

(A) Plasma Mean Temperature ( $T$ )

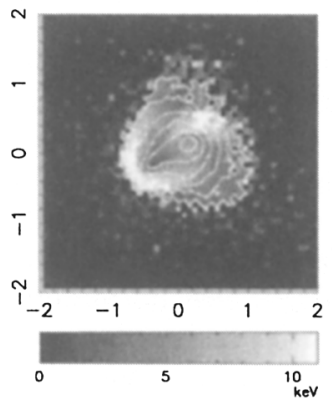

(B) Electron Temperature $\left(T_{e}\right)$

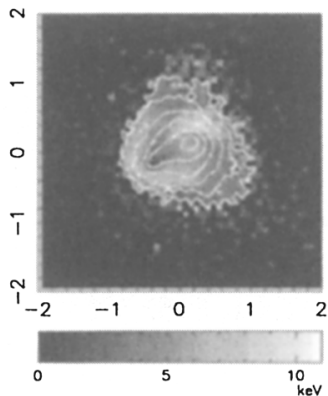

(C) Normalaized Temperature $\left(T_{e} / T\right)$

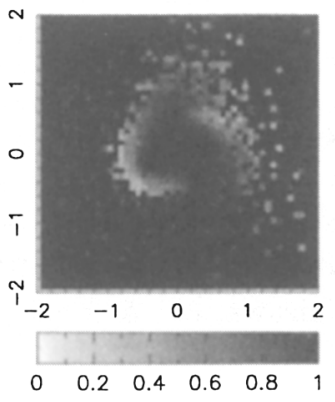

Figure 1. Snapshots of plasma mean temperature $(T)$ distribution (A), electron temperature $\left(T_{e}\right)$ one $(\mathrm{B})$, and normalized electron temperature $\left(T_{e} / T\right)$ one $(\mathrm{C})$ 

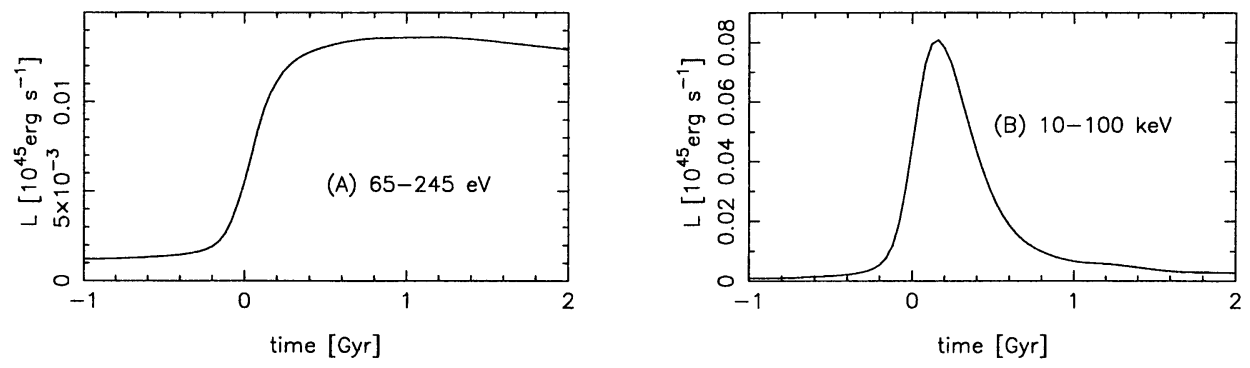

Figure 2. Time evolution of non-thermal emission: (A) EUV band $(65-245 \mathrm{eV})$ and $(\mathrm{B})$ hard X-ray band (10-100 keV)

\section{Results}

First, we calculate dynamical evolution of merging clusters of galaxies, explicitly considering the relaxation process between ions and electrons. We assume that artificial viscous heating is effective only for ions and that Coulomb coupling is considered in the relaxation process. Figure 1 shows snapshots of plasma mean temperature $(T)$, electron temperature $\left(T_{e}\right)$, and normalized electron temperature $\left(T_{e} / T\right)$ distribution of 1:4 merger $0.25 \mathrm{Gyr}$ after the most contracting epoch. Significant temperature difference between ions and electrons can be seen especially around the shocks. Note that we can estimate only electron temperature through current X-ray observations though dynamical state of ICM is related to plasma mean temperature.

Second, we calculate evolution of non-thermal emission from relativistic electrons accelerated around the shock fronts. We assume that $5 \%$ of viscous energy is transported to non-thermal electrons. Inverse Compton scattering of CMB photons, synchrotron losses, and Coulomb losses are considered for electron cooling. Figure 2 shows time evolution of non-thermal emission due to inverse Compton scattering of CMB photons during cluster merger. Hard $\mathrm{X}$-ray is luminous only while signatures of merging events are clearly seen in the thermal intracluster medium. On the other hand, extreme ultraviolet emission is still luminous after the system has relaxed.

\section{References}

Takizawa, M. 1999, ApJ, 520, 514

Takizawa, M. 2000, ApJ, 532, 183

Takizawa, M., \& Naito, T. 2000, ApJ, 535, 586 